\title{
THE DIAGNOSIS AND MANAGEMENT OF PULMONARY EMBOLISM
}

\author{
D. W. BARRITT, M.D., M.R.C.P. \\ Consultant Physician, United Bristol Hospitals.
}

\begin{abstract}
EMBOLISM of the pulmonary arteries presents in one of three main ways. Most dramatic is acute failure of the circulation. A large clot obstructs more than half of the cross-section of the pulmonary tree at the bifurcation of the main stem or the hila of both lungs. More commonly it is the development of an infarct at the base of one or both lungs that gives evidence of an embolism which has lodged some hours before. Rarely, repeated small emboli, which do not separately give rise to symptoms or signs, may lead to mounting pulmonary hypertension and chronic right heart failure.
\end{abstract}

\section{The Attendant Circumstances}

Pulmonary embolism is distincly uncommon in patients who have previously been healthy and fully mobile but attention was drawn to this possibility by Homan's (1943) report of 11 such cases. Of the 120 cases of pulmonary embolism reported by Short (1952), three were well and fully mobile at the time of their first symptom.

Any patient confined to bed for a few days may develop the disease. Especially at risk are those ill with heart disease (Carlotti, Hardy, Linton and White, 1947; Short, 1952; Byrne, 1955), neoplasms (Trousseau, 1877; Sproul 1938), injuries to the bones of the legs (Sevitt and Gallagher, 1959), recent operations (Barker Nygaard, Walters and Priestley, 1940) or thrombophlebitis (Byrne, 1955). Although almost every patient with pulmonary embolism or pulmonary infarction has a source of thrombus formation in the venous system of the abdomen or legs, the condition will be diagnosed on the evidence in the heart or lungs. In two necropsy studies, McLachlin and Paterson (1951) found venous thrombosis in all of 19 cases of pulmonary embolism and in the series of Sevitt and Gallagher (1959) 32 of 38 patients with venous thrombosis had pulmonary embolism. Clinical methods of detecting venous thrombosis are, however, imperfect and in the absence of signs in the legs the absence of extensive thrombosis cannot be assumed. Thus, in Sevitt's patients, 9 of 17 who died of pulmonary embolism were without clinical evidence of thrombosis.
In a clinical study of 72 patients with puts. monary embolism reported by Barritt and Jordan (1961), 32 were found to have tendef ness of the calf on squeezing, 20 œdema of the leg, 10 superficial thrombophlebitis, $1 \bar{\phi}$ varicose veins and 16 no abnormal signs. Clinical evidence of a possible source in the leg maj therefore, be taken as a confirmatory sign of pulmonary embolism but in its absence $n \underline{\underline{\theta}}$ conclusions can be drawn.

Evidence of Obstruction to the Pulmonary Tres

Massive embolism of the pulmonary arter may be followed by immediate loss of cons sciousness and death within minutes. The patient is usually cyanosed and the pulse rapio and of very low volume. If the patient survives, evidence of severe circulatory obstruction will persist and the following are the principat clinical features:

Breathlessness and Restlessness. Anx accompanies breathlessness which may sisi itself with the least movement about the beck This immediate breathlessness is not due to pleural pain but indicates a profound disturb ance of ventilation. Orthopnœa is unusual. $\stackrel{2}{\rightleftarrows}$

Faintness. With low levels of blood pressure there may be feelings of faintness or loss of consciousness on sitting up. Faintness in the early period of getting out of bed after oper: ations should be viewed with particular suspicion.

Central Chest Pain. Discomfort in the centre of the chest is indistinguishable from the pain of myocardial infarction. Radiation to the arms or jaw is uncommon but the pain may be severe and occasionally may last for hours.

Tachycardia. A rise in heart rate is a ver frequent accompaniment of pulmonary embaj lism. In cases where the first symptoms afe those of the development of an infarct, pulse charts frequently show a sharp rise in heaft rate some hours before the onset of pleurat pain, and this serves to date the arrival of the embolus. Paroxysmal arrhythmias also occuf. Short (1952) considered that five of his 120 patients developed atrial fibrillation as a resuto of embolism and one other had ventricula tachycardia. Barritt and Jordan (1961) cors firmed the onset of atrial fibrillation in fou 


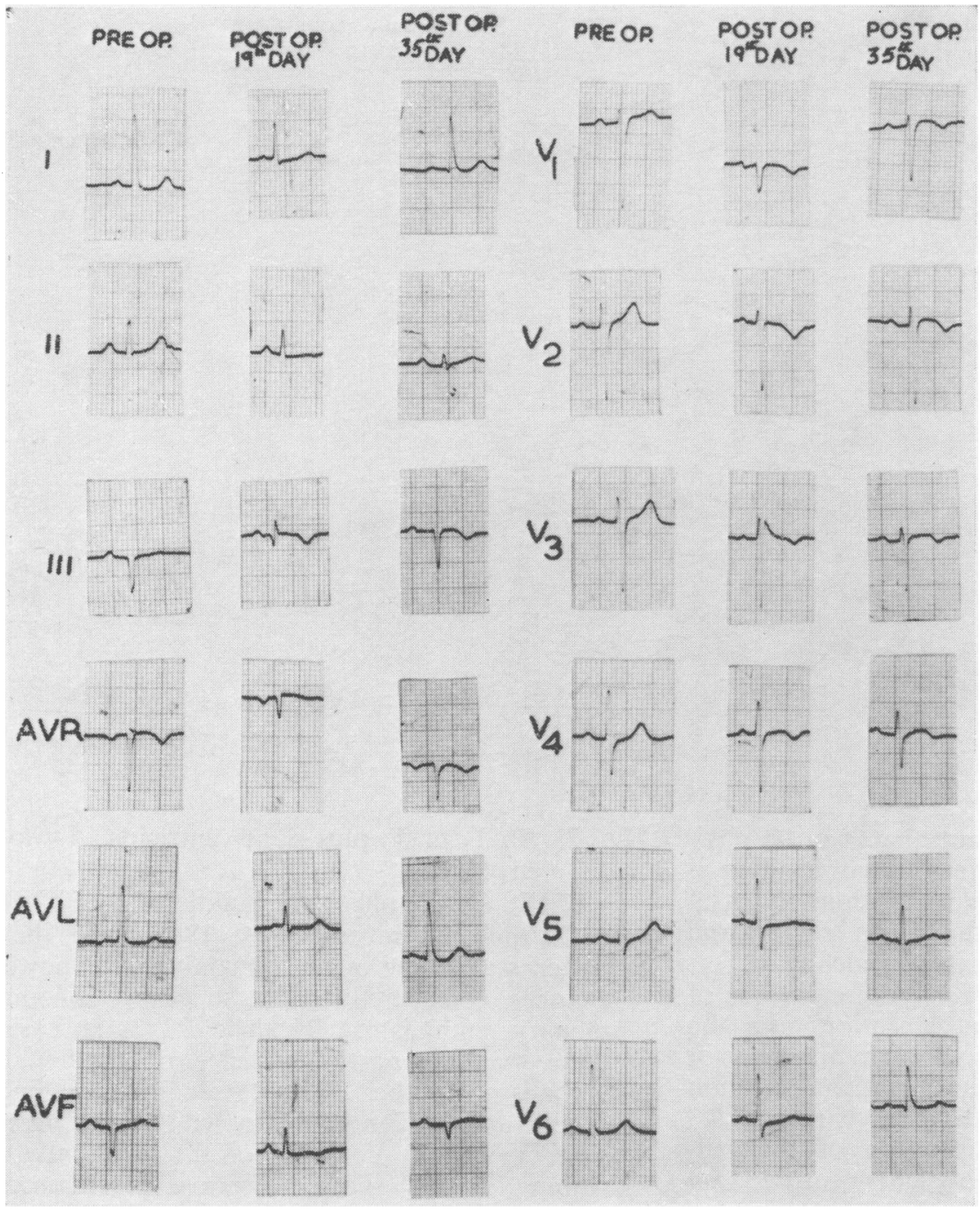

Fig. 1.-Successive Electrocardiograms taken before operation, after the occurrence of massive embolism (Post-op. 19th day) and after 16 days' a n icoagulant treatment.

of their 72 patients and paroxysmal supraventricular tachycardia in a fifth. The onset of atrial fibrillation during the course of heart disease should, therefore, lead to thought being given to the possibility that pulmonary embolism has occurred.

Hypotension. An abrupt fall in blood pressure can be taken to indicate a reduced cardiac output. Occasionally, the diagnosis is first suggested when routine records of blood pressure show a fall in systolic pressure to less than $100 \mathrm{~mm}$. Hg. As in other low output states an obvious fall in systolic pressure during inspiration may be a feature but pulsus para- doxus on palpation is rare. With the low blood pressure and poor volume pulse the skin is cold and clammy.

Cyanosis. Arterial oxygen desaturation is very difficult to detect in the patient with a cold skin, but cyanosis of the tongue and mucous membranes is occasionally obvious and arterial puncture will then confirm the desaturation. An increase in the dead space within the lung and disturbance of ventilation perfusion ratio, together with diminished compliance are likely to be the causative mechanisms.

Raised right atrial pressure. Obstruction to the outflow from the right ventricle leads to 


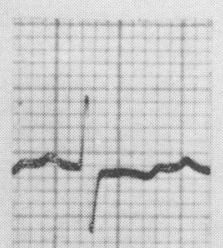

I

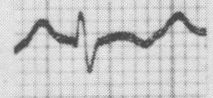

x

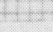

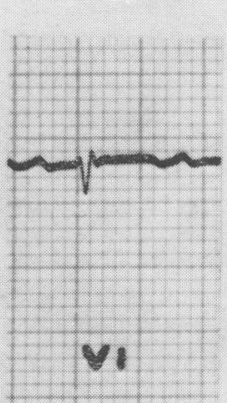
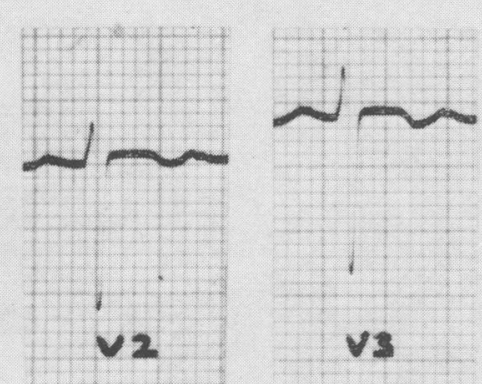
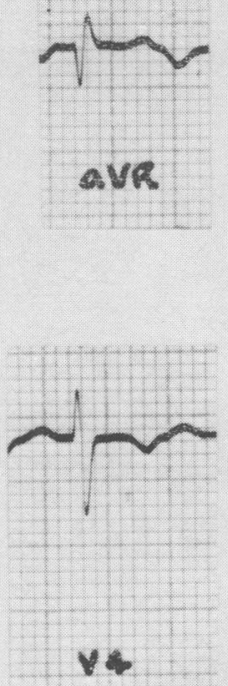

aVR

IT.
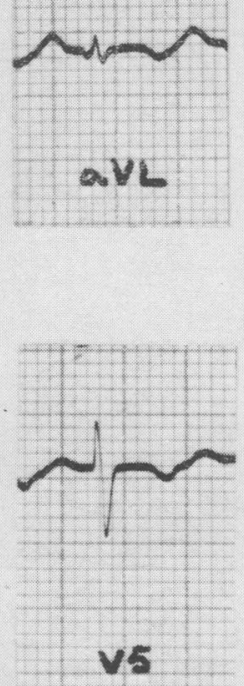

aVL

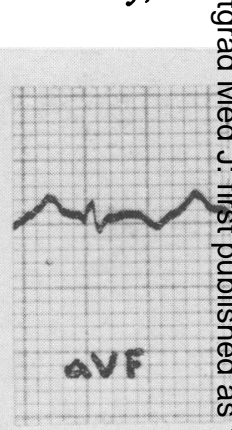

Fig. 2.-See Text.

a rise in right atrial pressure that may be obvious in the neck. In others, breathlessness is accompanied by such large variations in intrathoracic pressure, that the mean right atrial pressure cannot be estimated clinically.

Auscultation of the Heart. Auscultation of the heart gives little informaation. In the presence of tachycardia gallop rhythm may be heard. In a small number of patients, friction sounds are present for short periods at the base of the heart. Abrupt distension of the pulmonary artery or of the right ventricle are probably responsible.

\section{The Electrocardiogram}

Characteristic changes can be expected in the electrocardiogram of patients with massive embolism of the pulmonary arteries. These consist of a shift to the right of the electrical axis of the heart with clockwise rotation and $\mathrm{T}$-wave changes in right ventricular leads. Cutforth and Oram (1958) analysed records from 50 cases of pulmonary embolism, of which 28 showed evidence of a fall in blood pressure or a rise in venous pressure (severe cases) and 22 neither feature (mild cases). These authors considered the following three electrocardiographic patterns to be diagnostic of pulmonary embolism:

1. $S_{1}, Q_{3}, T_{3}$ plus right ventricular $T$-wave inversion.
2. $\mathrm{S}_{1}, \mathrm{~T}_{3}$ or $\mathrm{T}_{3}$ plus right ventricular $\mathrm{T}$ inversion.

3. $\mathrm{S}_{1}, \mathrm{Q}_{3}, \mathrm{~T}_{3}$ plus right bundle branch blocs.

Of their 28 severe cases, 18 showed the patterns and nine of the remaining ten show changes considered to be suggestive of put monary embolism. Of their 22 mild cases, there was diagnostic evidence of pulmonapy embolism in the electrocardiogram in hati. Barritt and Jordan (1961) noted T-wave invession in $V_{1}$ and $V_{2}$ in 14 of their patients, $\overrightarrow{\theta f}$ whom 11 also showed $\mathrm{T}$-wave inversion leads II or III or aVf. Transient right bundi़e branch block was present in a further foọr patients. Of these 18, there was a fall in blood pressure and other evidence of massige embolism in 16. Changes in the electricad position of the heart were present in others whilst of 12 patients with normal ECGS none had a fall in blood pressure. Obvious changes in the electrocardiogram, therefore, age likely in severe cases and suggestive evidenee may strengthen the diagnosis in those with leas circulatory disturbance.

In a small number of acutely ill patients with low blood pressure and high venous pressure the electrocardiogram may be less helpfu. Medd and McBrien (1962) cite patients wi massive pulmonary embolism with S-T depres sion or T-wave changes in left precordial lea@s 
which suggest the presence of coronary artery disease. Figure 2 shows the electrocardiogram in a woman of 40 years whose first symptom was a brief attack of effort syncope whilst on holiday. Very high venous pressure with tachycardia, small volume pulse and pulsus paradoxus suggested the diagnosis of cardiac tamponade. Shortly after attempted aspiration of the pericardium she died and at necropsy massive, repeated embolism of the pulmonary arteries was found.

\section{Evidence of Pulmonary Infarction}

When thrombus becomes tightly packed in a branch of the pulmonary artery, symptoms and signs of pulmonary infarction usually follow. This is more likely to occur when the embolus passes beyond the bifurcation into one or both branches towards the periphery of the lung. Only the lower lobes are usually affected and one may assume that this is because blood flow to the upper lobe is extremely small at rest in the upright position (Dollery, Hugh-Jones, Matthews, 1962).

\section{Pleural Pain-Friction and Effusion}

Pleural pain is the commonest symptom to draw attention to pulmonary embolism. It may be slight or severe and on one or both sides. A friction rub may or may not accompany it. In patients whose embolism is dated by the onset of faintness, breathlessnes or tachycardia, pleural pain may follow some hours later. A pleural effusion may develop. The fluid is usually bloodstained.

\section{Cough and Hamoptysis}

Cough, with the production of a small quantity of sputum, is frequently present. Hæmoptysis, which varied in amount from a speck of blood on one occasion only, to heavy staining of the sputum for many days, was present in 31 of 72 of Barritt and Jordan's patients. Breathlessness and Cyanosis

In the presence of pulmonary infarction, dypnœa is most commonly the result of pleural pain. The pain is avoided by taking shallow, rapid breaths. More severe breathlessness points to a high degree of circulatory obstruction. Cyanosis is usually inconspicuous except in the presence of extensive bilateral infarction. Physical Signs in the Chest

Breathing is usually quiet but increased respiratory variation in venous pressure observed at the root of the neck may point to diminished lung compliance.

Percussion of the chest usually confirms the presence of a lung lesion because resonance is impaired at the affected base. The loss of resonance rarely amounts to stony dullness. In contrast to the findings with consolidation of lobar pneumonia, breath sounds and voice sounds are diminished over an infarct. Bronchial breathing is a rare finding. The most frequent auscultatory sign is the presence of rales. Rales are rarely absent in pulmonary infarction, and are present at both bases in about half the cases, thus pointing to the presence of clots in both lungs.

\section{Fever and Jaundice}

Fever is an almost invariable accompaniment of pulmonary infarction. Characteristically, fever is present at the time of onset of pleural pain or hæmoptysis. With effective anticoagulant therapy and without the addition of antibiotic agents, fever soon disappears. Jaundice is much less frequent, especially in the absence of congestive heart failure.

\section{The Chest Radiograph}

Although the patient cannot be taken to the $\mathrm{X}$-ray Department, and despite breathlessness and pain at the time of the examination, changes in the chest radiograph will usually be found at the first examination in the ward. Short (1951) described such changes in 85 of 94 patients examined. The abnormalities were infarct shadows in 88 per cent, pleurisy in 56 per cent, elevation of the diaphragm in 39 per cent, and multiple infarcts in 43 per cent. These positive radiological changes were usually present within 12 hours of infarction. A negative chest X-ray should not weigh heavily against the diagnosis of pulmonary embolism, particularly if the film is of poor quality. Embolism without infarction may be present and here the radiological changes may be much less conspicuous. Shapiro and Rigler (1948) considered the characteristic abnormalities in embolism without infarction to be increased radio-translucency and ischæmia of the involved pulmonary segment, with abrupt termination of the embolised pulmonary artery.

\section{Pulmonary Function Tests}

Obstruction of a major branch of the pulmonary artery must necessarily cause an important disturbance of pulmonary function. Perfusion of the affected lung segment will cease, ventilation of the unperfused lung will continue and when infarction occurs the inflammatory process will disturb the lung further. Robin, Forkner, Bromberg, Croteau and Travis (1960) discussed the alveolar gas exchange in pulmonary embolism. They list three major components of disturbance: arterial oxygen desaturation, hyper-ventilation, and the 


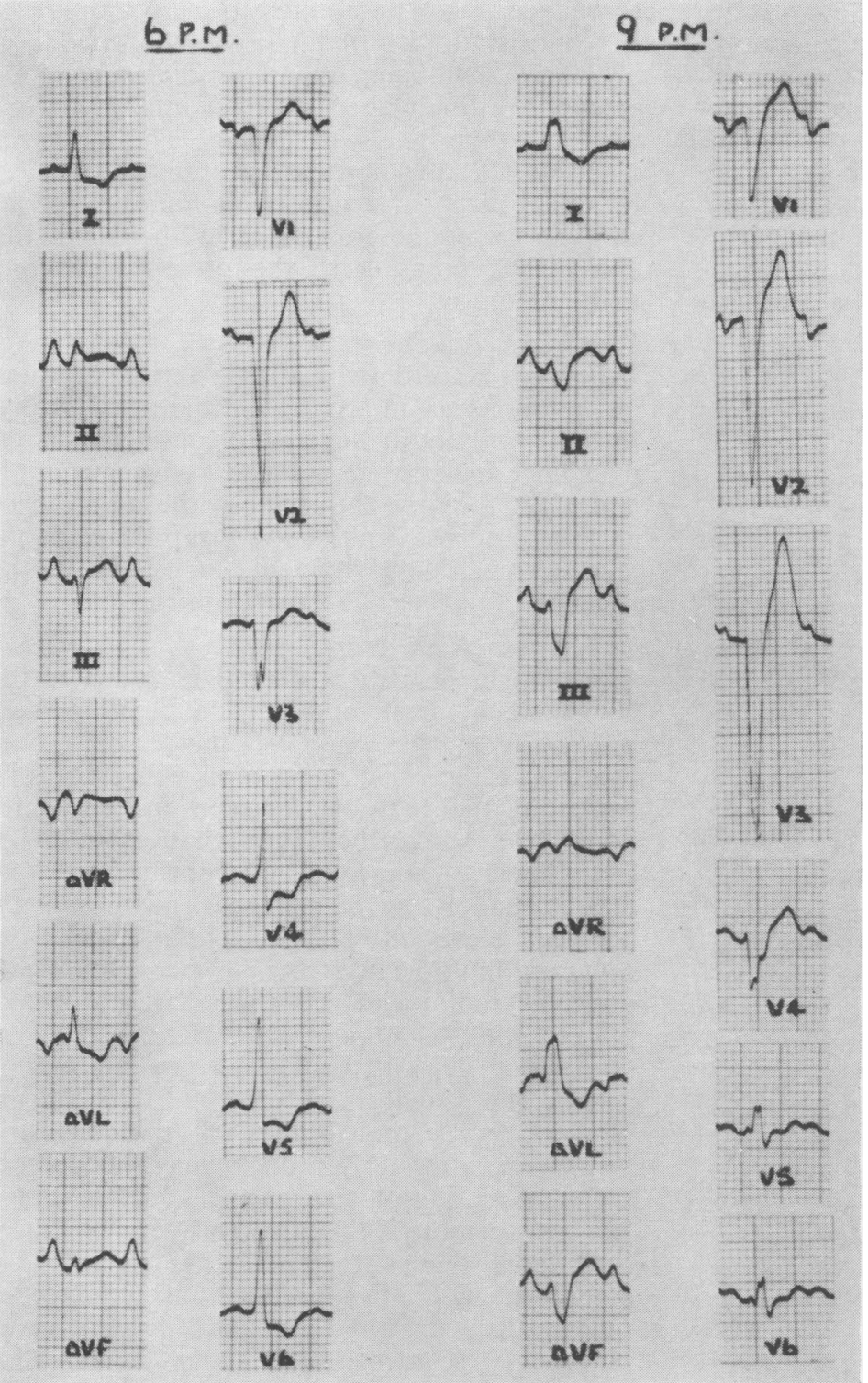

FIG. 3.-Successive tracings with interval of three hours in in elderly patient with extremely hig( venous pressure but no orthopnœa Pulmonary embolism was suspectef and consideration was given to surgical exploration. Progressive changes of left bundle branch block suggested myocardial infarction anf necropsy confirmed the diagnosis $\frac{}{<}$

development of differences between the $\mathrm{CO}_{2}$ tension of arterial blood and the end-tidal air as a result of dilution of alveolar air by newly-formed dead space. Measurement of the arterial-end tidal difference is clearly not an easy technique in an ill and breathless patient, and others have found difficulty in using this as a diagnostic test.

\section{Angiocardiography}

Possible use of angiocardiographic techniques in the acutely ill patient are considered subsequently when differential diagnosis is discussed.

Differential Diagnosis of Massive Pulmonary Infarction

When the physician is faced with a cardio vascular emergency in a patient with very low cardiac output, high venous pressure an central chest discomfort, the usual differen tial diagnosis is between massive pulmonar embolism and myocardial infarction. The dise tinction is usually not difficult for evidence of incipient pulmonary œedema together with the onset of an injury current in the electro cardiogram make the diagnosis of myocardia infarction. In the absence of these two features 
in the early hours of the emergency the circumstances of the attack may weight the scales in one direction or another. Clear evidence of venous disease of the legs suggests pulmonary embolism, and a fleck of blood stained sputum in the absence of wet lungs is confirmatory. Again, electrocardiography will usually bring confidence to the diagnosis of embolism. If the patient's condition is not deteriorating, reliance should be placed on serial electrocardiograms at frequent intervals (Fig. 3). An early rise in serum glutamic oxalacetic transaminase favours muscle necrosis from myocardial infarction.

It may be felt that the differential diagnosis is barely of practical importance as treatment of these two conditions is so similar. There will, however, be occasions when consideration is given to the idea of surgical removal of an embolus and, under these circumstances, diagnosis must be certain. There may be a place here for more adequate X-ray visualisation of the disturbance in the pulmonary vascular tree. High quality plain films of the hila of the lungs will repay close study. The possibility of the bedside use of contrast radiography needs to be further explored. Robb and Steinberg (1939) claimed that accurate studies could be made of arterial and venous patterns of the lung by injection of contrast medium into an arm vein with the patient sitting before a casette. Williams and Wilcox (1963) have recently made a study using this technique in 50 patients with pulmonary embolism. All the patients tolerated the procedure well but few details are given of the severity of their illness or the type of apparatus used. Mention is made of the possibility of this approach to the problem of the patient in whom surgical exploration is considered. Proof of major obstruction to vessels and localisation of the main areas of embolism might then be considered a necessary preliminary to operation.

When the emergency presents with paroxysmal arrhythmia differential diagnosis is likely to be more difficult. Treatment of the arrhythmia will take priority. The possibility of pulmonary embolism should always be considered when arrhythmia occurs in the course of heart disease of all types. The distinction from cardiac tamponade will not arise frequently. Where doubt exists a diagnostic aspiration is essential but one hopes that exploration of the pracordium with ultrasound recording may display or exclude a pericardial effusion without the use of a needle.
Pulmonary infarction must usually be distinguished from pneumonia especially in the post-operative period. No single feature is in itself decisive with the exception of the diagnostic electrocardiographic change which will be present in the minority of cases when a lung lesion is prominent. Physical signs of venous thrombosis will be present in the majority, and it is the combination of venous thrombosis and an appropriate pulmonary iesion that points to the diagnosis most frequently. Difficulties arise when there are no abnormal signs in the legs. Pneumonia will then often be diagnosed and antibiotics prescribed. The majority of patients will recover with these measures, but there will often be surprises as the late development of leg signs or recurrence of chest troubles, or even fatal embolism correct the diagnosis.

Where doubt exists response to anticoagulant therapy will sometimes give diagnostic help. With the infusion of heparin, fever may vanish within hours and not recur.

Repeated Minor Emboli leading to Progressive Pulmonary Hypertension

With the widespread recognition of the value of anticoagulant therapy in curing venous thrombo-embolism, repeated major embolisation leading to progressive right heart failure is a rare condition. A small group of patients exist, however, who exhibit progressive pulmonary artery obstruction who have minimal clinical evidence or no clinical evidence at all of pulmonary embolism or infarction. This condition appears in previously healthy people who have not been confined to bed. Goodwin, Harrison and Wilcken (1963) described 11 such patients and Wilhelmsen, Selander, Soderholm, Paulin, Varnauskas and Werko (1963) and a further 10. There is little except evidence of major filling defects in the pulmonary arteriogram, to distinguish this condition from so called primary pulmonary hypertension. Thus, the patients present with breathlessness of effort, effort syncope, chest pain and hæmoptysis. The electrocardiogram shows right ventricular dominance and catheterisation confirms the pulmonary hypertension. Goodwin lays great stress on the necessity for early recognition of this condition as successful arrest of the condition with anticoagulant treatment can be hoped for only before pulmonary hypertension becomes established. Slight effort dyspnoa with hyperventilation at rest is the clue. Under these circumstances arterial-end tidal $\mathrm{CO}_{2}$ 
gradients may well be demonstrated and arterial oxygen desaturation on effort is likely.

Management of Venous Thrombo-Embolism

It would be inappropriate to discuss the treatment of pulmonary embolism without considering prophylaxis first. Attempts to diminish the incidence of pulmonary embolism in hospital by systematically allowing patients to get out of bed at an earlier stage of their illness have met with little success. This is probably because at an early stage of recovery from illness or operations the level of activity is of necessity very low and nothing is gained by exchanging bed rest for chair rest.

This has led to a study of the use of anticoagulant drugs given prophylactically to patients who are especially at risk. Sevitt and Gallagher (1959) in their classical study compared 150 elderly patients with fractured hips (control series) with a similar number who were given Phenindione routinely soon after admission to hospital. This prophylactic use of Phenindione resulted in complete freedom from pulmonary embolism in the period of treatment, whereas in the control series 18 per cent of patients developed pulmonary embolism and 10 per cent died of it. There was also a great saving in venous thrombosis in the legs. In a somewhat similar study, Chalmers, Marks, Bottomley and Lloyd (1960) tested the use of prophylactic anticoagulant therapy in selected groups of gynæcological and obstetric patients. In all they tested 1877 patients in a five year period and made a comparison with a preceding five year period when prophylactic anticoagulants had not been used. They too found an impressive reduction in venous thrombosis and pulmonary embolism in treated patients. A strong case can, therefore. be made for the use of anticoagulants given prophylactically to patients who are at special risk.

A different approach to the problem has been suggested by Sharnoff, Kass and Mistica (1962) on the basis of a state of increased blood coagulability as demonstrated by LeeWhite clotting time values. They administered Heparin subcutaneously before operation and before mobilising the patient in the postoperative period. Their recommendations were not supported by a sufficiently extensive clinical trial to prove the value of this type of therapy.

\section{Treatment of Massive Embolism with Circula-} tory Failure

As in other circulatory or respiratory emergencies, reassurance of the patient is of prime importance. The relief of anxiety by confidence of the physician, aided whe्षfe necessary by pain relieving drugs, will do much to economise respiratory effort aind diminish heart rate.

When the pulmonary artery becomes neagly completely obstructed by a large clot, imme ate therapy can only be directed to sustainifor the contractile force of the right ventricle. \&o long as sufficient blood can be forced post the obstruction to maintain an impoverished circulation it can be expected that the embali will tend to be packed into smaller branches, thus allowing full perfusion of some areas $8 \mathrm{~s}$ the lung. In the critical period, maintenare of arterial oxygen saturation and perfusionn pressure in the coronary circulation, and contiol of the heart rate are likely to assist the ventricle.

Arterial oxygen saturation will certainty fall under these circumstances and high concentrations of oxygen should be given by face mask.

With low central aortic pressure, corona $\frac{\bar{c}}{\sqrt{r} y}$ blood flow will fall. Mephentermine sulphate, metaraminol or infusions of angiotension \&r noradrenaline should be used if the systotic blood pressure falls below $90 \mathrm{mmHg}$. Inf the presence of signs of tachycardia or paroxysmml arrhythmia rapid digitalisation is indicated.

Heparin should be given at once to d\$8courage the formation of fresh thrombus $\frac{0}{\mathrm{at}}$ sites where blood flow is obstructed by the embolism.

If the patient survives long enough for the measures to be put into operation then he expect to recover with continued medical therapy. Of the 53 patients in Barritt agd Jordan's (1960) treated series, none died of pulmonary embolism who survived long enough to receive the first injection of Heparin.

This favourable outcome cannot, however, be expected in every case and some patients may survive for a number of hours with $\cong \mathrm{a}$ falling blood pressure before they die. Wh these exceptional circumstances the question of surgical removal of the emboli may now be rationally considered. Reports of success'ful removal of clot have been given by Hampson, Milne and Small (1961) wo operated at normal temperature without persfusion, by Alison, Dunnill and Marshall (1960) operating after surface cooling of their patiegt to $29^{\circ} \mathrm{C}$ and by Cooley, Beall and Alexandor (1961) using cardiopulmonary bypass.

A planned operation of this type shoufd 
certainly only be carried out if deterioration continues in spite of intensive medical treatment.

In patients who die within minutes of their embolism, attempts to restore the circulation by continued external cardiac compression and artificial ventilation may succeed from time to time. Such methods are well directed to the younger patient stricken unexpectedly by a fatal circulatory disturbance.

\section{Management of Pulmonary Infarction}

The question of more prolonged therapy of pulmonary embolism can be considered under this heading. Additional features which call for further mention are pleural pain and fever. Pethidine is a suitable drug for the relief of the pain of pulmonary infarction and 50-100 mg. by intramuscular injection can be given at once and repeated if need be.

Unless there are strong contraincications, anticoagulant treatment will always be given. Recent operations are rarely a great hazard. In patients with a history suggesting active peptic ulceration it may be preferable to use Heparin as the only anticoagulant drug throughout the course of treatment. Heparin may be given by intermittent intravenous injection or by continuous infusion for many days. The latter method has the advantage that the normal clotting mechanism is restored very rapidly if the infusion is terminated. Usually, an oral anticoagulant will be administered at the same time as Heparin is started so that Heparin is discontinued after $36-48$ hours as the oral anticoagulant takes over. Phenindione has been used most extensively in Britain in the last decade but occasional reports of serious toxic reactions affecting kidney, liver and bone marrow have led some authors to question its suitability (Holman). High levels of dosage are called for and one stage Quick times should be prolonged to $2 \frac{1}{2}-3$ times control times.

Antibiotic therapy is usually unnecessary for fever will fall soon after the infarct begins to heal, in the same way as in myocardial infarction. Pulmonary infarcts can, however, become infected with organisms from the upper respiratory tract and this possibility should be borne in mind. Anticoagulant therapy should be continued until clinical evidence of infarction has been cleared and evidence of active disease in the leg has subsided. By this time, clearing of the changes on X-ray of the chest will usually be evident and in most cases a three weeks' course of treatment is adequate. If there are still signs of infarction, either on clinical or radiological grounds at the end of three weeks, then therapy should be prolonged. Occasionally, there will be recurrence of signs in the chest or leg and then a second course of treatment will be called for and it should be prolonged for four to six weeks. Occasionally, continued treatment for 6-12 months may be necessitated by recurring evidence of thrombo-embo'ism. Ligation of veins will very rarely be indicated.

\section{Thrombolytic Therapy}

Fibrinolytic enzymes may find a place in the treatment of pulmonary embolism. It is clear from the natural history of the condition that large clots are spontaneously lysed in the pulmonary artery and it is logical, therefore, to attempt to assist this process. To this end, activators of the Plasminogen-Plasmin reaction, particularly Streptokinase, have been used. Preparations of Streptokinase carry a risk of febrile reactions and in the critical state of the circulation after massive embolism this hazard contra-indicates the use of this type of therapy at the present time. Results with Heparin are so good that new remedies should not be used until more evidence is available of their superiority. Caution concerning the use of streptokinase has been expressed by McNicol, Douglas and Bayley (1962) who made a detailed study of Streptokinase infusions in patients with vascular occlusions.

\section{Repeated Embolism with Pulmonary Hyper- tension}

This uncommon condition calls for the institution of anticoagulant treatment at the earliest possible moment and the treatment should be pressed for months, or years, until all clinical and laboratory evidence of the disease has disapeared.

In the presence of heart failure, digitalis should be added but if this becomes necessary, arrest of the changes in the pulmonary artery cannot be expected.

\section{REFERENCES}

Allison, P. R., Dunill, M. S. T., Marshall, R. (1960): Pulmonary Embolism. Thorax, $15,273$.

Barker, N. W., Nygaard, K. K., Walters, W., Priestley, J. T. (1940): A Statistical Study of Post-Operative Venous Thrombosis and Pulmonary Embolism. Proc. Mayo. Clin., 15, 769.

BARRITT, D. W., JoRDAN, S. C. (1960): Anticoagulant Drugs in the Treatment of Pulmonary Embolism. A Controlled Trial. Lancet i, 1309. 
Barritt, D. W., Jordan, S. C. (1961): Clinical Features of Pulmonary Embolism. Lancet, i, 729.

BYRNe, J. J. (1955): Phlebitis. New Engl. J. Med., 253, 579.

CARlotti, J., HARDY, B. I., Linton, R. R., WhITE, P. D. (1947): Pulmonary Embolism in Medical Patier. J. Amer. med. Ass., 134, 1447.

Chalmers, D. G., Marks, J., Bottomley, J. E., Lloyd, O. (1960) i Post-Operative Prophylactic Anticoagulants. A 5-year Study in an Obstetric and Gynæcological Unit. Lancet, ii, 220.

Cooley, A. D., Beall, A. C., Alexander, J. K. (1961): Acute Massive Pulmonary Embolism. Successfall Surgical Treatment Using Temporary Cardio-pulmonary Bypass. J. Amer. med. Ass., 177, 283.

CUTFORTH, R. H., ORAM, S. (1958): The Electrocardiogram in Pulmonary Embolism. Brit. Heart J., 20, 委.

Dollery, C. T., Hugh-Jones, P., Mathews, C. M. E. (1962): Use of Radio-Active Xenon for Studje of Regional Lung Function. Brit. med. J., 2, 1006.

Goodwin, J. F., Harrison, C. V., WILCKEN, D. E. L. (1963): Obliterative Pulmonary Hypertension a@d Thrombo-Embolism. Brit. med. J., 1, 701 and 707.

Hampson, J., Milne, A. C., Small, W. P. (1961): The Surgical Treatment of Pulmonary Embolism. Lancênt, ii, 402.

Holman, V. Personal Communication, and in Press.

Homan, J. (1943): Pulmonary Embolism due to Quiet Venous Thrombosis and Simulating Cardiac a $\overrightarrow{\overrightarrow{a g d}}$ Pulmonary Disease. New Engl. J. Med., 229, 309.

Mclachlin, J., Paterson, J. C. (1951): Some Basic Observations on Venous Thrombosis and Pulmon Embolism. Surg. Gynec. Obstet., 93, 1.

MCNicol, G. P., Douglas, A. S., BAYLEY, C. (1962): Experience with Streptokinase Infusions. Lancet $\frac{3}{11}$, 1297.

MedD, W. E., McBrien, D.J. (1962): Acute Pulmonary Embolism with Electrocardiographic Changes Mimicking Coronary Artery Disease. Lancet, ii, 944,

RobB, G. P., Steinberg, E. (1939): Visualisation of the Chambers of the Heart. Amer. J. Roentgenol, 1 , 1 .

Robin, E. D., Forkner, E. C., Bromberg, P. A., Croteau, J. R., and Travis, D. M. (1960): Alveotar Gas Exchange in Clinical Pulmonary Embolism. New Engl. J. Med., 262, 283.

SevitT, S., Gallagher, N. G. (1959)): Prevention of Venous Thrombosis and Pulmonary Embolism th Injured Patients. Lancet, ii, 981.

Shapiro, R., Rigler, L. G. (1948): Pulmonary Embolism without Infarction. Amer. J. Roentgenol, 460 .

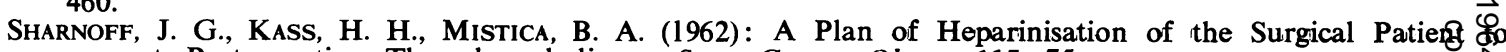
prevent Postoperative Thromboembolism. Surg. Gynec. Obstet, 115, 75.

SHORT, D. S. (1951): A Radiological Study of Pulmonary Infarction. Amer. J. Med., 44, 233.
SHORT, D. S. (1952): A Survey of Pulmonary Embolism in a General Hospital. Brit. Med. J., 1, 790.9

SPROUL, E. E. (1938): Carcinoma and Venous Thrombosis: The Frequency of Association of Carciroma in the Body or Tail of the Pancreas with Multiple Venous Thrombosis. Amer. J. Cancer, 34, 566.

Trousseau, A. (1877): Clin. Med. Hotel-Dieu. Paris: Bailliére.

Wilhelmsen, L., Selander, S., Soderholm, B., Paulin, S., Varnauskas, E., Werko, L. (1963): Recurreghit Pulmonary Embolism. Medicine (Baltimore), 42, 335.

Williams, J. R., Wilcox, W. C. (1963): Pulmonary Embolism. Roentgenographic and Angiographic Comsiderations. Amer. J. Roentgenol, 89, 333. 